Case Report

COpen Access

CrossMark

\title{
Severe fatal pulmonary hypertension in newborn with idiopathic arterial calcification
}

\begin{abstract}
Idiopathic congenital calcification of the great vessels is a rare genetic disorder affecting the infants during first months of live causing fatal complications ${ }^{1-3}$ due to generalized calcification of the moderate to large vessels leading to decrease elasticity and occlusion of these vessels. ${ }^{4}$ We prescribe 2 days old boy with respiratory distress and mild central cyanosis, his echocardiography reveal a calcified aorta and pulmonary arteries with sever pulmonary hypertension that was diagnosed later as idiopathic congenital arterial calcification of infancy.
\end{abstract}

Volume 2 Issue 2 - 2015

\author{
Mahmoud Alsoufi \\ Consultant Pediatric Cardiologist, Al Qassimi Hospital, United \\ Arab Emirates
}

Correspondence: Mahmoud Alsoufi, Consultant Pediatric Cardiologist, Al Qassimi Hospital-Sharjah, United Arab Emirates, Tel 0097I-553405476,Email dr.m.soufi@hotmail.com

\section{Introduction}

This rare and unusual disease is sometimes easily too misdiagnosed, it is a genetically inherited autosomal recessive disorder characterized by extensive arterial calcification and stenosis of medium to large arteries causing stiffness and decrease of elasticity and sometimes occlusion. Pathologically, there is calcium deposition in the internal elastic membrane of arteries associated with fibrous thickening of the intima causing narrowing of the lumen of the affected vessels. This pathophysiology is the main cause of pulmonary and occasionally systemic hypertension. Accurate diagnosis is important since follow up with genetic counseling is mandatory. Only few cases were reported previously in the literature as a case report and our case is one of them with very early presentation and the first case reported in our area.

\section{Case report}

2Few hours old baby boy full term $3.1 \mathrm{Kg}$, delivered by cesarean section with poor Apgar score 6 and 8 in first and fifth minute respectively. Baby was noticed also to have generalized edema including the umbilical cord with abdominal distension due to mild ascites (picture of hydropsfetalis) he was shifted immediatelyto NICU and connected to ventilator due to respiratory failure.

a. By physical examination: $\mathrm{HR}=145 / \mathrm{min}, \mathrm{RR}$ : on ventilator , $\mathrm{BP}=55 / 30 \mathrm{mmHg}$, week peripheral pulse, normal $\mathrm{S} 1+\mathrm{S} 2+$ soft systolic murmur $2 / 6$ at left lower sterna border, liver is palpable 3 $\mathrm{cm}$ below right costal margin.

b. Blood investigation revealed: Metabolic acidosis, impaired liver functions, impaired renal function, Normal electrolytes and no signs of sepsis.

c. Past medical history: The mother was not screened during this pregnancy but she had 2 abortions for unknown reasons during her third trimester and one child 2years old alive and healthy.

d. His chest and abdominal x-ray showed: Cardiomegaly with calcified abdominal aorta (Figure 1).

e. Echocardiography showed: dilated right and left heart, impaired systolic functions, sever pulmonary hypertension (almost systemic) and calcified great vessels (aorta + pulmonary) (Figure 2).

f. Abdominal ultrasound: Mild to moderate generalized hepatomegaly with significant calcification of descending aorta and great branches (Figure $3 \& 4$ ). According to that the Patient was covered empirically by antibiotic with supportive therapy for pulmonary hypertension (inotropes + High frequency ventilation and silfenafil) and investigated for the possible causes of calcified great vessels. All hormonal investigations and TORCH infection screen were negative. Chromosomal analysis showed normal study so genetic sample were sent for specific study. In spite of aggressive supportive therapy, he did not show any clinical improvement and deteriorated gradually and ended by cardiorespiratory arrest and death declared at day 12 of life.
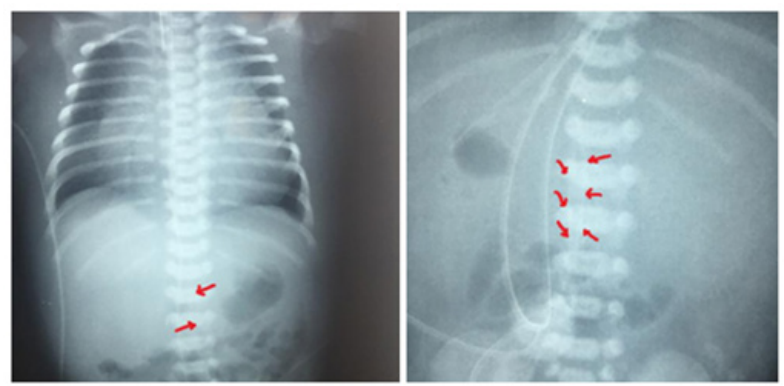

Figure I Cardiomegaly with calcified aorta (arrows).

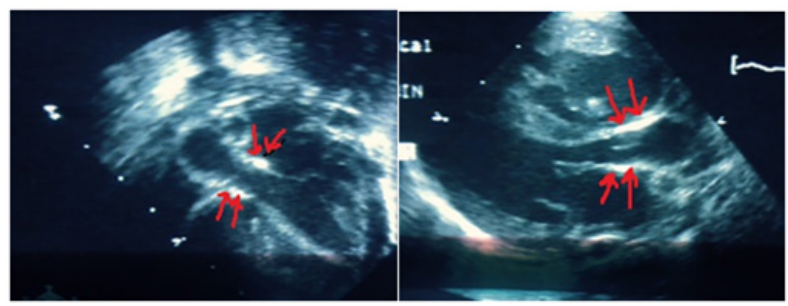

Figure 2 Calcified Aorta.

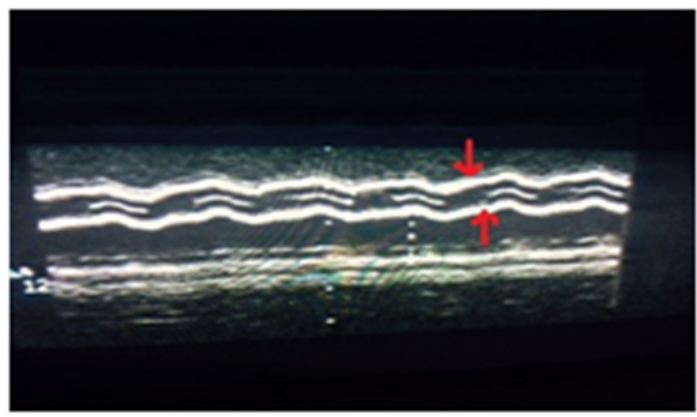

Figure 3a Calcified aorta in M-mode. 


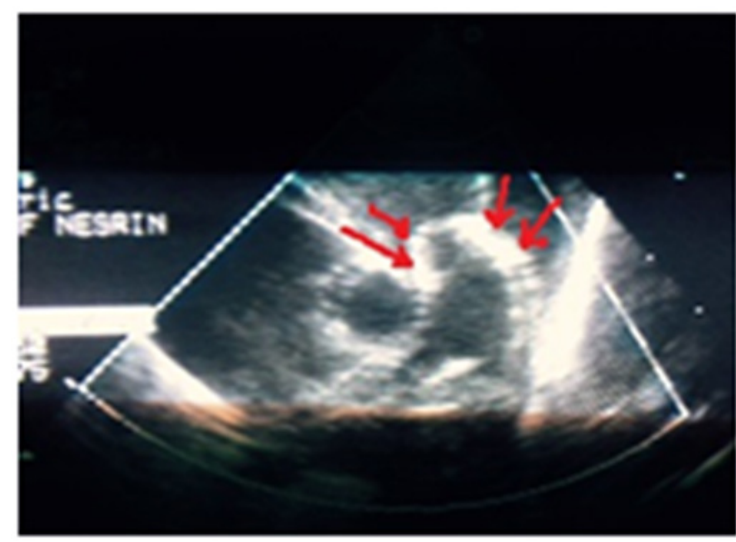

Figure 3b Calcified pulmonary.

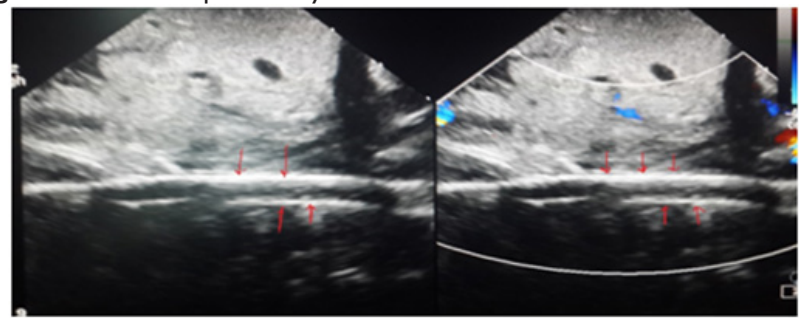

Figure 4 Abdominal ultrasound.

\section{Discussion}

Idiopathic arterial calcification can present very early in life even parental, prenatal or early postnatal and it affects the medium and large vessels all over the body causing loss of compliance and elasticity with narrowing and even occlusion of this blood vessel ${ }^{5}$ with different degree of systemic and pulmonary hypertension. ${ }^{5,6}$ This rare disease of unknown etiology might be fatal antenatal or early in life due to sever pulmonary or systemic hypertension with signs of hydropsfetalis in the fetus or heart and multi organ failure postnatal. Death from this disease also might be related to occlude vessels especially the coronaries arteries causing myocardial infarction early in life. ${ }^{7}$ There is a different cause of pulmonary hypertension in the newborns and idiopathic arterial calcification still one of the very rare causes and usually associated with severe form of pulmonary and occasionally systemic hypertension. ${ }^{8}$

The final diagnosis of this disease depends on multi issues: positive maternal history about? Form of hydropsfetalis, family history of abortions in the last trimester of pregnancy; ${ }^{9}$ sever form of pulmonary hypertension and its complications with unknown causes early in life and finally supported evidence of calcified great vessels on echocardiography and ultrasound studies. ${ }^{6}$

Genetic consultation is still essential for the final diagnosis and screening of other family members also well recommended to detect the silent cases. Since no a life case with this disease is present, we do not know who this kind of disease can affect the life quality and span of those patients. The trial of treatment by diphosphonate is mentioned in the literatures with some case reports of total recovery after few years. ${ }^{10-13}$

\section{Conclusion}

In our case, it was the first case in our area to discover this disease with very early presentation and dramatically progression, so the patient was treated by supportive therapy mainly for sever pulmonary hypertension and the idea of diphosphonate treatment was discussed one day before his death but still we recommend it as an option for the treatment for those patients.

\section{Acknowledgments}

None.

\section{Conflicts of interest}

Author declares there are no conflicts of interest.

\section{References}

1. Meradji M, de Villeneuve VH, Huber J, et al. Idiopathic infantile arterial calcification in siblings: radiologic diagnosis and successful treatment. $J$ Pediatr. 1978;92(3):401-405.

2. Moran $\mathrm{Jj}$, Steiner Gc. Idiopathic arterial calcification in a 5-year-old child. A case report. Am J Clin Pathol. 1962;37:521-526.

3. Rangaswami N, Chaudhary S, Flinn G, et al. Idiopathic linear calcification of the ascending aorta in an adolescent. Am J Dis Child. 1979;133(8):860-861.

4. Maayan $\mathrm{C}$, Peleg $\mathrm{O}$, Eyal $\mathrm{F}$, et al. Idiopathic infantile arterial calcification: a case report and review of the literature. Eur J Pediatr. 1984;142(3):211-215.

5. Anderson KA, Burbach JA, Fenton LJ, et al. Idiopathic arterial calcification of infancy in newborn siblings with unusual light and electron microscopic manifestations. Arch Pathol Lab Med 1985;109(9):838-842.

6. Milner LS, Heitner R, Thomson PD, et al. Hypertension as the major problem of idiopathic arterial calcification of infancy. $J$ Pediatr. 1984;105(6):934-938.

7. Lussier-Lazaroff J, Fletcher BD. Idiopathic infantile arterial calcification: roentgen diagnosis of a rare cause of coronary artery occlusion. Pediatr Radiol. 1973;1(4):224-228.

8. Sholler GF, Yu JS, Bale PM, et al. Generalized arterial calcification of infancy: three case reports, including spontaneous regression with longterm survival. J Pediatr. 1984;105(2):257-260.

9. Witzleben CL. Idiopathic infantile arterial calcification--a misnomer? Am J Cardiol. 1970;26(3):305-309.

10. Retbi JM, Casasoprana A, Gabilan JC, et al. Idiopathic arterial calcification in infancy. A case report. Eur J Pediatr. 1978;129(1):55-60.

11. Bird T. Idiopathic arterial calcification in infancy. Arch Dis Child. 1974;49(2):82-89.

12. Hunt AC, Leys DG. Generalized arterial calcification in infancy. Brit Med J. 1957;1(5015):385-386.

13. Meradji M, de Villeneuve VH, Huber J, et al. Idiopathic infantile arterial calcification in siblings: radiologic diagnosis and successful treatment. $J$ Pediatr . 1978;92(3):401-405. 